\title{
REMOÇÃO DE CHUMBO(II) EM SISTEMAS CONTÍNUOS POR CARVÃO ATIVADO COM VAPOR
}

\author{
Cristiane Imenes de Campos Bueno Zanin e Flávia Camargo Alves Figueiredo \\ Faculdade de Engenharia Química, Universidade Estadual de Campinas, CP 6066, 13083-970 Campinas - SP, Brasil \\ Wagner Alves Carvalho* \\ Centro de Ciências Naturais e Humanas, Universidade Federal do ABC, Rua Santa Adélia, 166, 09210-170 Santo André - SP, Brasil
}

Recebido em 20/10/08; aceito em 26/5/09; publicado na web 6/11/09

\begin{abstract}
LEAD(II) REMOVAL IN CONTINOUS SYSTEMS BY VAPOR ACTIVATED CARBON. Fixed-bed column studies were undertaken to evaluate the performance of a commercial Brazilian activated carbon in removing $\mathrm{Pb}$ (II) from aqueous environment. Breakthrough points were found out for the metal adsorption by varying different operating parameters like feed concentrations (10 and $\left.20 \mathrm{mg} \mathrm{L}^{-1}\right)$ and bed heights $(0.5,1.5$ and $2.8 \mathrm{~cm}$ ). A good agreement was observed between the experimental data and the values predicted by the bed depth service time (BDST) model. Regeneration of the exhausted columns was possible with $\mathrm{HCl}$, and the adsorption capacity was maintained after three adsorption-desorption cycles.
\end{abstract}

Keywords: activated carbon; lead(II); column adsorption.

\section{INTRODUÇÃO}

Os metais potencialmente tóxicos são largamente utilizados na indústria, particularmente na laminação de metais. Alguns deles também estão presentes em determinados pesticidas e até mesmo em medicamentos. São ainda usados em pigmentos, esmaltes, tintas e corantes. Em virtude deste vasto espectro de utilidades, os metais aportam em sistemas aquáticos por várias fontes e espécies diferentes. ${ }^{1,2}$

Dentre os metais pesados mais preocupantes, por sua alta toxicidade e larga utilização, cita-se o chumbo. ${ }^{3,4}$ Geralmente, o chumbo não constitui um problema ambiental até que venha a se dissolver e produzir a forma iônica, cuja espécie mais estável é a divalente, encontrada naturalmente na forma de galena $(\mathrm{PbS}) .{ }^{5}$ A contaminação humana por chumbo é relatada desde a antiguidade. Em 2001, no Brasil, noticiou-se largamente a contaminação por chumbo das águas do Rio Ribeira de Iguape, nos estados de São Paulo e Paraná, e de moradores da região, devido a atividades desde 1920 de uma mineradora de chumbo que encerrou suas atividades em 1996, mas manteve rejeitos nas margens do rio. ${ }^{6} \mathrm{O}$ limite máximo de descarte de chumbo em corpos receptores permitido pela legislação é de 0,5 $\mathrm{mg} \mathrm{L}^{-1}$ (Conselho Nacional de Meio Ambiente - CONAMA, Resolução $\left.n^{\circ} 357 / 2005\right)$.

Dentre os vários processos de tratamento para efluentes contaminados por metais, é mais frequente a precipitação de óxidos hidratados pela adição de hidróxido de cálcio ou de soda cáustica. Embora este método seja relativamente simples e barato, tem como principais desvantagens: a geração de um grande volume de lodo a ser descartado no final do processo; dependendo do metal e das condições de precipitação, é impossível atingir a concentração limite de poluentes permitida por lei; são necessários longos tempos de decantação e/ou filtração das partículas gelatinosas de hidróxidos dos metais pesados, mesmo na presença de coagulantes e floculantes próprios; ocorre um aumento considerável da alcalinidade do efluente. ${ }^{7}$ Além disso, esse processo não se mostra eficaz para a descontaminação de grandes volumes de efluentes contendo metais pesados, principalmente em baixas concentrações, devido à baixa eficiência operacional e aos elevados custos de remoção resultantes do processo. ${ }^{8}$ Portanto, existe a necessidade de um tratamento estratégico, que seja simples

\footnotetext{
*e-mail: wagner.carvalho@ufabc.edu.br
}

e robusto, direcionado a recursos viáveis e adequados a problemas locais. ${ }^{9,10} \mathrm{O}$ uso de materiais naturais, abundantes e de baixo custo, é uma alternativa bastante explorada. ${ }^{11,12}$ Mesmo com pequenas modificações estruturais, estes materiais ainda demonstram viabilidade de uso, uma vez que estas alterações levam a aumentos consideráveis na capacidade de retenção. ${ }^{13,14} \mathrm{O}$ carvão ativado figura como potencial candidato, principalmente quando consideramos que o Brasil é um país com elevado potencial de produção de carvão, tanto a partir de madeiras de reflorestamento quanto de resíduos agrícolas. ${ }^{15}$

Em trabalho recente, avaliamos a capacidade de retenção de chumbo(II) presente em meio aquoso por dois carvões ativados brasileiros, produtos comerciais fornecidos pela Brascarbo Agroindustrial Ltda. (Paraná, Brasil). ${ }^{16}$ Os resultados indicaram que o equilíbrio foi atingido nos primeiros $5 \mathrm{~min}$ de contato entre o adsorvente e o efluente, com uma capacidade máxima de adsorção superior a 69 $\mathrm{mg} \mathrm{g}^{-1}$ para o carvão ativado com vapor (CarboActiv V-plus). Estes resultados, aliados à capacidade de dessorção do metal adsorvido, permitiram prever a possibilidade de utilização deste carvão em sistemas contínuos, nos quais o material é testado em colunas de adsorção, efetuando-se ciclos de adsorção/dessorção consecutivos. Embora os testes em batelada forneçam informações úteis para a remoção de contaminantes específicos, os estudos com colunas de adsorção correspondem à maioria das aplicações práticas do processo de adsorção no tratamento de efluentes. ${ }^{17}$

Neste trabalho, avaliamos a capacidade de retenção de chumbo(II) presente em meio aquoso pelo carvão CarboActiv V-plus, obtendo a curva de ruptura no processo de adsorção em colunas, bem como a realização de ciclos de adsorção/dessorção.

\section{PARTE EXPERIMENTAL}

O carvão comercial utilizado como adsorvente foi fornecido pela Brascarbo Agroindustrial Ltda. (Paraná, Brasil) e identificado como CarboActiv V-plus. O carvão foi utilizado sem qualquer tratamento prévio. Trata-se de um carvão ativado com vapor d'água a $800^{\circ} \mathrm{C}$. Segundo laudo fornecido pelo fabricante, a granulometria deste carvão corresponde a 97,6\% do sólido passante na malha 325 mesh, enquanto o seu número de iodo é de $975 \mathrm{mg} \mathrm{g}^{-1}$ e o teor de cinzas, de 2,4\%. Uma caracterização complementar deste material foi apresentada em trabalho anterior. ${ }^{16} \mathrm{O}$ carvão possui comportamento anfótero, com ponto de carga zero em $\mathrm{pH}$ 
10,5. Dentre os grupos funcionais ácidos de superfície, tem-se ampla predominância de grupos carboxílicos. A área superficial é de $872 \mathrm{~m}^{2}$ $\mathrm{g}^{-1}$, com raio médio dos microporos em torno de $6,0 \AA$.

\section{Avaliação da adsorção de $\mathbf{P b}($ II) em leito fixo}

Os estudos de adsorção foram realizados em colunas com $1 \mathrm{~cm}$ de diâmetro e $5 \mathrm{~cm}$ de comprimento. Tipicamente foi utilizada uma massa do carvão em pó igual a 0,3 g, o que corresponde a uma profundidade de $0,5 \mathrm{~cm}$. Posteriormente, foi avaliado o comportamento de colunas contendo 0,5 e $0,7 \mathrm{~g}$ de carvão, o que corresponde às profundidades de 1,5 e $2,8 \mathrm{~cm}$, respectivamente.

Uma bomba peristáltica Ismatec, com uma vazão volumétrica de $1 \mathrm{~mL} \mathrm{~min}^{-1}\left(\sim 0,19 \mathrm{~m}^{3} \mathrm{~m}^{-2} \mathrm{~h}^{-1}\right)$ possibilitou o controle da passagem das soluções de $\mathrm{Pb}$ (II) pela coluna. As concentrações iniciais das soluções foram de 100, 20 e $10 \mathrm{mg} \mathrm{L}^{-1}$. Alíquotas foram recolhidas em certos intervalos de tempo e filtradas em filtro Millex-HV com membrana de PVDF, 0,45 um de diâmetro de poros e $13 \mathrm{~mm}$ de diâmetro, acoplado a uma seringa de vidro. A quantificação do metal em solução foi feita por espectrofotometria de absorção atômica (EAA), em um equipamento Perkin Elmer modelo Analyst 300, utilizando-se curva analítica preparada a partir de diluições de uma solução de $\mathrm{Pb}(\mathrm{II})$ contendo $1000 \mathrm{mg} \mathrm{L}^{-1}$ (Titrisol ${ }^{\circledR}$, Merck). Os resultados demonstraram uma resposta linear da absorbância em função da concentração na faixa de 1,0 a 20,0 $\mathrm{mg} \mathrm{L}^{-1}$, utilizada nas quantificações posteriores.

Os testes foram realizados à temperatura ambiente $\left(\sim 25^{\circ} \mathrm{C}\right)$ e o $\mathrm{pH}$ da solução ficou em torno de 5,5. Um pHmetro digital Analyser modelo $300 \mathrm{M}$ foi usado nas medidas de $\mathrm{pH}$.

\section{Avaliação da dessorção de $\mathrm{Pb}(\mathrm{II})$ em leito fixo e reciclagem da coluna}

Após a exaustão do carvão ativado por $\mathrm{Pb}(\mathrm{II})$ foi necessário regenerar o material adsorvente para uso posterior. Sendo assim, foi avaliada a dessorção da coluna utilizando uma solução de $\mathrm{HCl} 1$ mol L ${ }^{-1}$. Alíquotas foram recolhidas em certos intervalos de tempo, filtradas e quantificadas. Após o término do processo a coluna foi lavada com água deionizada. Ciclos de adsorção/dessorção foram realizados para duas concentrações inicias, 10 e $20 \mathrm{mg} \mathrm{L}^{-1}$, e para as três massas de carvão avaliadas.

\section{RESULTADOS E DISCUSSÃO}

\section{Avaliação inicial dos processos de adsorção e dessorção}

Uma avaliação inicial, realizada com uma solução de $\mathrm{Pb}$ (II) de concentração $100 \mathrm{mg} \mathrm{L}^{-1}$, permitiu verificar o comportamento do sistema tanto em relação à adsorção quanto à possibilidade de dessorção do $\mathrm{Pb}(\mathrm{II})$. Foi possível observar que a coluna pode operar durante cerca de $40 \mathrm{~h}$, considerando-se um ponto de exaustão do leito em C/ $\mathrm{C}_{0}$ igual a 0,9 . O ponto de ruptura (breakpoint), estabelecido quando $\mathrm{C} / \mathrm{C}_{0}$ for igual a 0,1 , foi atingido em cerca de $3 \mathrm{~h}$ de operação. Já a dessorção mediada por tratamento com solução de $\mathrm{HCl}$ mostrou-se bastante eficiente, uma vez que o leito pode ser recuperado em um intervalo de tempo inferior ao de exaustão da coluna (após $6 \mathrm{~h}$ de tratamento $\mathrm{C} / \mathrm{C}_{0}$ era igual a 0,2 ).

Em semelhança ao que ocorreu nos testes em batelada, ${ }^{16} \mathrm{o}$ uso de $\mathrm{HCl} 1 \mathrm{~mol} \mathrm{~L}^{-1}$ permite a dessorção praticamente total do $\mathrm{Pb}$ (II) adsorvido no carvão. O equilíbrio foi atingido com o uso de cerca de $156 \mathrm{~mL}$ da solução de $\mathrm{HCl}$.

Os resultados indicaram que a coluna apresenta elevada eficiência na retenção do $\mathrm{Pb}(\mathrm{II})$. A capacidade de retenção do carvão pode ser calculada a partir da curva de ruptura de $\left(\mathrm{C}_{0}-\mathrm{Ci}\right)$ em função do tempo, onde $\mathrm{C}_{0}$ é a concentração inicial de $\mathrm{Pb}$ (II) no efluente e $\mathrm{C}_{\mathrm{i}}$ é a concentração em equilíbrio após o tempo t. A área abaixo da curva de ruptura corresponde à medida da capacidade de retenção e é dada pela Equação 1:

$$
C L=V \int_{0}^{T}\left(C_{0}-C_{i}\right) d t
$$

onde CL é a capacidade de retenção do leito, $\mathrm{V}$ é a vazão de efluente e T é o tempo necessário para a exaustão total do adsorvente.

Os cálculos indicaram que CL corresponde a $10,4 \mathrm{mg} \mathrm{g}^{-1}$. Este valor é muito próximo do valor identificado para a mesma concentração inicial de metal nos testes em batelada $\left(12,7 \mathrm{mg} \mathrm{g}^{-1}\right) .{ }^{16}$ Portanto, os resultados demonstraram que o procedimento utilizado para os testes em colunas permitiu um aproveitamento adequado da capacidade de adsorção. Certamente valores inferiores àqueles obtidos no processo em batelada já seriam esperados. Quando em batelada, a suspensão é continuamente agitada e uma interação adequada entre as fases sólida e líquida é favorecida. Por outro lado, nas colunas as partículas do adsorvente estão em contato íntimo, o que dificulta a interação sólidosolução. Além disso, existe a possibilidade de formação de canais preferenciais para a percolação do líquido, o que resulta em um tempo de residência na coluna menor e uma interação menos efetiva entre o sólido e o metal. Diante destes resultados, promovemos um teste semelhante, substituindo a solução contendo $100 \mathrm{mg} \mathrm{L}^{-1}$ de $\mathrm{Pb}(\mathrm{II})$ por soluções mais diluídas, com 10 e $20 \mathrm{mg} \mathrm{L}^{-1}$, o que corresponde a uma situação mais realística de uso prático de colunas de adsorção.

\section{Influência da massa de carvão}

As curvas de ruptura obtidas com diversas massas de carvão e uma concentração inicial de $\mathrm{Pb}(\mathrm{II})$ de $10 \mathrm{mg} \mathrm{L}^{-1}$ são apresentadas na Figura 1. De maneira semelhante, foram construídas as curvas de ruptura para uma concentração inicial de $20 \mathrm{mg} \mathrm{L}^{-1}$ (Figura 2).

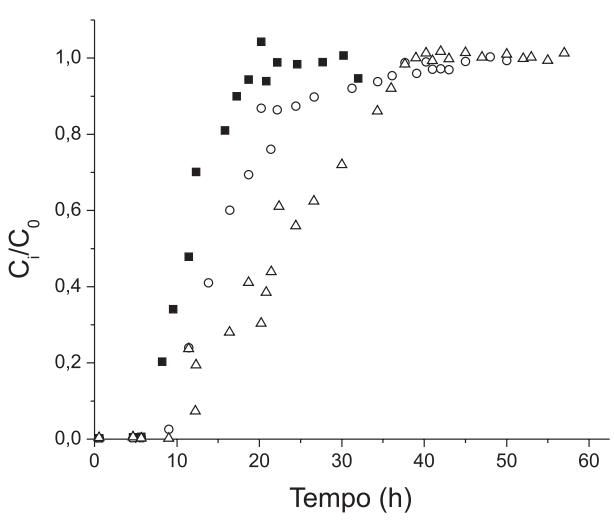

Figura 1. Curvas de ruptura para $\mathrm{Pb}(\mathrm{II}) 10 \mathrm{mg} \mathrm{L}^{-1}$ com diferentes quantidades

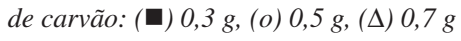

Os resultados relacionados aos pontos de exaustão e de ruptura estão resumidos na Tabela 1. É possível observar que as colunas podem operar durante intervalos de tempo elevados, atingindo $57 \mathrm{~h}$ até a exaustão para um sistema com $0,7 \mathrm{~g}$ de carvão. Nestas condições, o ponto de ruptura foi alcançado após $12 \mathrm{~h}$ de operação.

Vários modelos matemáticos têm sido desenvolvidos para uso em colunas de adsorção. ${ }^{18}$ Dentre os modelos abordados, o modelo que correlaciona profundidade da coluna versus tempo de operação (bed depth service time, BDST), baseado na Equação de Bohart-Adams ${ }^{19}$ é largamente utilizado. ${ }^{20,21} \mathrm{O}$ modelo de Bohart-Adams considera que o equilíbrio na coluna não é instantâneo, sendo que a velocidade de adsorção depende da fração de sítios de adsorção livres presente na superfície 


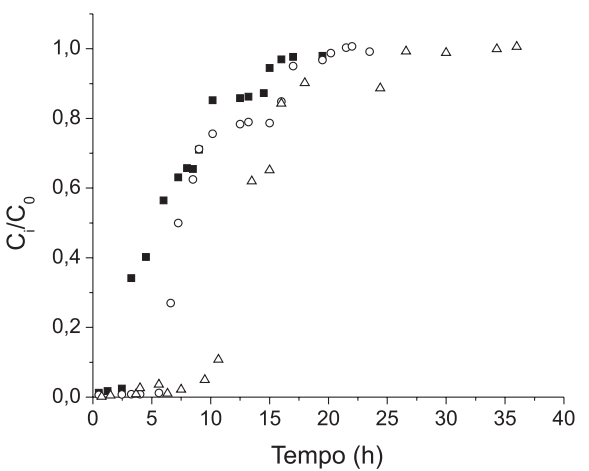

Figura 2. Curvas de ruptura para $\mathrm{Pb}$ (II) $20 \mathrm{mg} \mathrm{L}^{-1}$ com diferentes quantidades de carvão: (ロ) $0,3 \mathrm{~g}$, (o) $0,5 \mathrm{~g}$, ( $\Delta) 0,7 \mathrm{~g}$

Tabela 1. Pontos de exaustão e de ruptura em função da concentração inicial de $\mathrm{Pb}(\mathrm{II})$ e da massa de carvão

\begin{tabular}{lccc}
\hline $\begin{array}{l}\mathrm{C}_{0} \\
\left(\mathrm{mg} \mathrm{L}^{-1}\right)\end{array}$ & $\begin{array}{c}\text { Massa de carvão } \\
(\mathrm{g})\end{array}$ & $\begin{array}{c}\text { Ponto de Exaustão } \\
(\mathrm{h})\end{array}$ & $\begin{array}{c}\text { Ponto de Ruptura } \\
(\mathrm{h})\end{array}$ \\
\hline 10 & 0,3 & 32 & 7 \\
& 0,5 & 50 & 8 \\
& 0,7 & 57 & 12 \\
20 & 0,3 & 20 & 3 \\
& 0,5 & 24 & 4 \\
& 0,7 & 36 & 10 \\
\hline
\end{tabular}

do adsorvente. Este modelo pode ser representado pela Equação 2: ${ }^{19}$

$$
\ln \left(\frac{C_{0}}{C_{B}}-1\right)=\ln \left(e^{K N_{0}(X / V)}-1\right)-K C_{0} t
$$

onde $C_{0}$ é a concentração inicial do soluto $\left(\mathrm{mg} \mathrm{L}^{-1}\right), C_{B}$ é a concentração desejada do soluto no ponto de ruptura $\left(\mathrm{mg} \mathrm{L}^{-1}\right), K$ é uma constante da faixa de adsorção $\left(\mathrm{L} \mathrm{mg}^{-1} \mathrm{~h}^{-1}\right), N_{0}$ é a capacidade de adsorção $\left(\mathrm{mg} \mathrm{L}^{-1}\right), X$ é a profundidade da coluna $(\mathrm{cm}), V e ́$ a velocidade linear do fluxo de alimentação da coluna $\left(\mathrm{cm} \mathrm{h}^{-1}\right), t$ é o tempo de operação da coluna (h).

A forma da Equação de Bohart-Adams, mostrada na Equação 2 , pode ser usada para determinar o tempo de operação, $t$, de uma coluna com profundidade $X$, dados os valores de $N_{0}, C_{0}$ e $K$. Estes valores podem ser determinados pela operação de colunas, com uma velocidade de fluxo, $V$. Ajustando $t=0$ e resolvendo a Equação 2 para $X$ temos a Equação 3:

$X_{0}=\frac{V}{K N_{0}} \ln \left(\frac{C_{0}}{C_{B}}-1\right)$

onde $X_{0}$ representa a altura mínima da coluna necessária para produzir uma concentração de efluente $C_{B}$, também conhecida como altura crítica da coluna.

De acordo com Kumar e Bandyopadhyay, ${ }^{20}$ pelo menos nove testes individuais de coluna devem ser feitos para coletar dados suficientes e necessários para utilizar a abordagem de Bohart-Adams. Entretanto, Hutchin ${ }^{22}$ propôs uma modificação neste modelo, identificada pela sigla BDST, a qual requer apenas três testes operacionais de coluna. A Equação de Bohart-Adams pode ser reescrita como:

$t=a X+b$

onde

$$
\begin{aligned}
& a=\frac{N_{0}}{C_{0} V} \\
& b=-\frac{1}{K C_{0}} \ln \left(\frac{C_{0}}{C_{B}}-1\right)
\end{aligned}
$$

Portanto, os valores de $N_{0}$ e $K$ podem ser facilmente calculados a partir da equação da reta obtida, conforme Equações 5 e 6. Os dados das curvas de ruptura foram tratados de acordo com este modelo, objetivando avaliar o tempo de operação necessário para atingir um valor pré-determinado de concentração do efluente. Este valor foi definido como sendo o limite estabelecido para descartes contendo $\mathrm{Pb}$ (II), de acordo com a Resolução 357 do CONAMA (2005), que é de $0,5 \mathrm{mg} \mathrm{L}^{-1}$. A partir do modelo Bohart-Adams foram calculados os tempos teóricos previstos para saturação da coluna e para que fosse alcançado o valor limite da legislação ambiental. Os resultados foram comparados com os valores reais, observados em laboratório.

Além dos valores de $K$ e $N_{0}$, também é possível avaliar a altura mínima da coluna $\left(X_{0}\right)$ necessária para permitir uma concentração de efluente $C_{B}$ igual a $0,5 \mathrm{mg} \mathrm{L}^{-1}$, utilizando a Equação 3. Os parâmetros calculados a partir deste modelo estão relacionados na Tabela 2, enquanto uma comparação entre os tempos teóricos e práticos para se atingir uma determinada retenção é apresentada na Tabela 3.

Tabela 2. Parâmetros calculados a partir da Equação de Bohart-Adams

\begin{tabular}{lcccccc}
\hline $\begin{array}{l}\mathrm{C}_{0} \\
\left(\mathrm{mg} \mathrm{L}^{-1}\right)\end{array}$ & $\begin{array}{c}\text { Retenção } \\
(\%)\end{array}$ & $\begin{array}{c}\mathrm{a} \\
\left(\mathrm{h} \mathrm{m}^{-1}\right)\end{array}$ & $\begin{array}{c}\mathrm{b} \\
(\mathrm{h})\end{array}$ & $\begin{array}{c}\mathrm{N}_{0} \\
\left(\mathrm{mg} \mathrm{mL}^{-1}\right)\end{array}$ & $\begin{array}{c}\mathrm{K} \\
\left(\mathrm{L} \mathrm{h}^{-1} \mathrm{mg}^{-1}\right)\end{array}$ & $\mathrm{R}^{2}$ \\
\hline 10 & 10 & 4,25 & 2,330 & 3,24 & $-0,13$ & 0,968 \\
& 90 & 5,52 & 6,579 & 4,22 & $-0,04$ & 0,999 \\
20 & 10 & 2,781 & 0,518 & 4,25 & $-0,35$ & 0,871 \\
& 90 & 3,918 & 1,493 & 5,99 & $-0,12$ & 0,999 \\
\hline
\end{tabular}

Tabela 3. Tempos das curvas de ruptura observados e previstos com base nas constantes de Bohart-Adams

\begin{tabular}{lcccc}
\hline $\mathrm{C}_{0}\left(\mathrm{mg} \mathrm{L}^{-1}\right)$ & Retenção $(\%)$ & $\mathrm{X}(\mathrm{cm})$ & $\mathrm{t}_{\text {previsto }}(\mathrm{h})$ & $\mathrm{t}_{\text {observado }}(\mathrm{h})$ \\
\hline 10 & 10 & 1,0 & 6,6 & 6,0 \\
& & 1,5 & 8,7 & 9,5 \\
& & 2,8 & 14,2 & 12,0 \\
& & 1 & 12,1 & 12,0 \\
& & 1,5 & 14,9 & 15,0 \\
& & 2,8 & 22,0 & 22,0 \\
& \multirow{2}{*}{10} & 1,0 & 3,3 & 2,5 \\
& & 1,5 & 4,7 & 5,8 \\
& & 2,8 & 8,3 & 8,0 \\
& \multirow{2}{*}{90} & 1,0 & 5,4 & 5,5 \\
& & 1,5 & 7,4 & 7,3 \\
& & 2,8 & 12,5 & 12,5 \\
\hline
\end{tabular}

Podemos observar que os resultados obtidos experimentalmente estão de acordo com os valores previstos, o que comprova que o modelo utilizado é adequado para a previsão do comportamento da coluna, dentro da faixa de parâmetros estudados. Outras variáveis ainda poderiam ser consideradas. Por exemplo, variando-se a concentração inicial do metal ou a vazão do efluente é possível recalcular os coeficientes da curva conforme as Equações 7 e 8, respectivamente. ${ }^{8}$

$a_{n}=a_{o}\left(\frac{C_{o}}{C_{n}}\right)$ 


$$
b_{n}=b_{o}\left(\frac{C_{o}}{C_{n}}\left\lceil\frac{\ln \left(C_{n}-1\right)}{\ln \left(C_{0}-1\right)}\right]\right.
$$

A capacidade de retenção do carvão pode ser calculada com o uso da Equação 3. Os valores são apresentados na Figura 3 e mostram pouca variação entre si. De maneira geral, observa-se uma pequena redução na capacidade de retenção com o aumento da massa de carvão, o que provavelmente está relacionado a problemas difusionais que são mais intensos na medida em que as colunas ficam maiores. Neste caso, a probabilidade de criação de caminhos preferenciais aumenta e, até mesmo, o contato íntimo do carvão com a fase líquida fica dificultado.

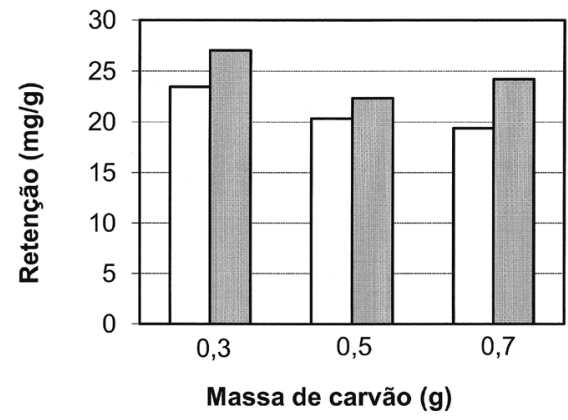

Figura 3. Resultados obtidos durante os cálculos de capacidade máxima de retenção para as concentrações iniciais estudadas: $10 \mathrm{mg} \mathrm{L}^{-1}$ (branco) e $20 \mathrm{mg} \mathrm{L}^{-1}$ (cinza)

\section{Avaliação da dessorção de $\mathrm{Pb}(\mathrm{II})$ em leito fixo}

As Figuras 4 e 5 indicam os resultados de dessorção obtidos para as concentrações iniciais de 10 e $20 \mathrm{mg} \mathrm{L}^{-1}$, respectivamente.

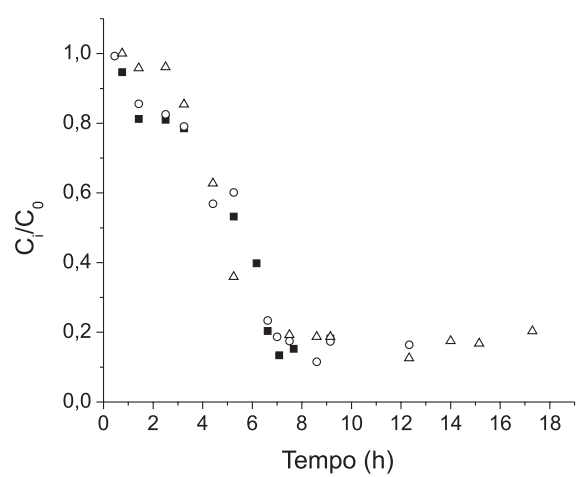

Figura 4. Primeira dessorção de Pb(II) para a concentração inicial de 10

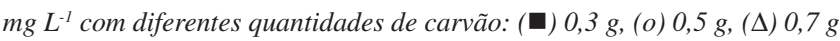

A dessorção mediada por tratamento com solução de $\mathrm{HCl}$ mostrou-se bastante eficiente, uma vez que o leito pode ser recuperado em um intervalo de tempo relativamente curto, tipicamente inferior ao tempo de operação da coluna observado durante o processo de adsorção. Para a concentração de $10 \mathrm{mg} \mathrm{L}^{-1}$, com 0,3 e $0,5 \mathrm{~g}$ de carvão, após 6,5 h de tratamento um valor de $\mathrm{C} / \mathrm{C}_{0}$ igual a 0,2 foi atingido, enquanto que para $0,7 \mathrm{~g}$ de carvão foram necessárias 7,5 h. Em relação à concentração de $20 \mathrm{mg} \mathrm{L}^{-1}$, para as duas massas inferiores de carvão foram necessárias $5 \mathrm{~h}$ de tratamento para se atingir o mesmo valor de $\mathrm{C} / \mathrm{C}_{0}$, enquanto que para $0,7 \mathrm{~g}$ foram necessárias 5,5 h. Em semelhança ao que ocorreu nos testes em batelada, ${ }^{16}$ o uso de $\mathrm{HCl} 1 \mathrm{~mol} \mathrm{~L}{ }^{-1}$ permitiu a dessorção pratica- mente total do $\mathrm{Pb}$ (II) adsorvido no carvão. Também foi possível observar que o processo de dessorção é pouco dependente da massa de carvão presente na coluna, bem como da concentração inicial empregada no processo.

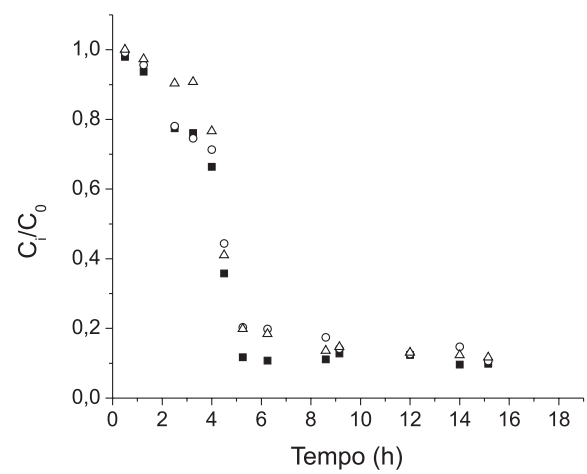

Figura 5. Primeira dessorção de $P b(I I)$ para a concentração inicial de 20

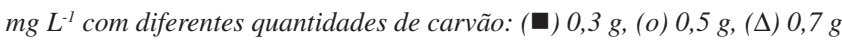

\section{Ciclos de adsorção-dessorção}

Com o intuito de estudar a eficiência do material adsorvente, tanto na recuperação como na regeneração do material, foram realizados três ciclos de adsorção-dessorção. A variação na capacidade de retenção ao longo dos ciclos é pequena, com valores inferiores a $1 \%$ de redução entre os ciclos. Este comportamento é observado de maneira semelhante para todas as massas de carvão e vazões estudadas e pode ser considerado como bastante satisfatório. Avaliando um sistema de remoção de $\mathrm{Pb}(\mathrm{II})$ por alumina em colunas de adsorção, Naiya et $_{\text {al. }}{ }^{23}$ identificaram uma redução de $13 \%$ na eficiência da coluna após os três ciclos acompanhados. Mesmo assim, os autores indicaram que este sistema apresenta um bom potencial para remoção em ciclos de adsorção-dessorção. Pehlivan et al. ${ }^{24}$ observaram que um sistema contínuo de adsorção de $\mathrm{Pb}$ (II) por biossorventes apresentou uma redução entre 20 e $25 \%$ após três ciclos de adsorção-dessorção. Uma elevada eficiência em ciclos foi observada por Sari e Tuzen ${ }^{25}$ na retenção de $\mathrm{Pb}(\mathrm{II})$ e $\mathrm{Cd}(\mathrm{II})$ em biossorventes, com apenas $2 \%$ de redução nos três primeiros ciclos e $10 \%$ em dez ciclos.

Portanto, o carvão CarboActiv V-plus utilizado neste trabalho demonstrou ser estável nas condições de uso, permitindo que tanto o processo de regeneração quanto os ciclos de adsorção-dessorção ocorram de modo eficiente.

\section{CONCLUSÕES}

O uso do carvão CarboActiv em processo de adsorção contínuo foi bastante satisfatório quando comparado com sistemas de adsorção semelhantes para o mesmo metal. Os resultados obtidos experimentalmente estão de acordo com os valores previstos pelo modelo utilizado, o que demonstra que a operação em coluna de adsorção com o carvão analisado leva a resultados práticos adequados, principalmente mediante o tratamento de soluções com baixa concentração. Já a dessorção com $\mathrm{HCl}$ se mostrou eficiente, uma vez que para ambas as concentrações de $\mathrm{Pb}(\mathrm{II})$ analisadas, o metal foi recuperado em um intervalo de tempo inferior àquele observado para exaustão da coluna. Os resultados obtidos para a capacidade máxima de retenção nos três ciclos de adsorçãodessorção foram bastante promissores, indicando que a coluna mantém a elevada capacidade de retenção de $\mathrm{Pb}(\mathrm{II})$ observada na primeira etapa de adsorção. 


\section{MATERIAL SUPLEMENTAR}

Estão disponíveis em http://quimicanova.sbq.org.br, na forma de arquivo PDF, com acesso livre: as curvas de ruptura obtidas com uma solução com concentração inicial de $\mathrm{Pb}$ (II) de $100 \mathrm{mg} \mathrm{L}^{-1}$, as curvas de profundidade da coluna em função do tempo de serviço e também as capacidades máximas de adsorção nos três ciclos de adsorção-dessorção estudados, para as concentrações iniciais de $\mathrm{Pb}$ (II) de 10 e $20 \mathrm{mg} \mathrm{L}^{-1}$ - Figuras $1 \mathrm{~S}$ a $6 \mathrm{~S}$.

\section{AGRADECIMENTOS}

Ao apoio financeiro concedido pelo CNPq (470458/04-6) e à Brascarbo Agroindustrial Ltda. que forneceu o carvão ativado utilizado como adsorvente neste trabalho. C. I. C. B. Zanin agradece à FAPESP pela bolsa concedida (05/04342-7)

\section{REFERÊNCIAS}

1. Manahan, S. E.; Environmental Science and Technology, Lewis Publishers: New York, 1997.

2. Gasselin, R. E.; Smith, R. P.; Hodge, H. C.; Chemical Toxicology of Commercial Products, Williams and Wilkins: London, 1984.

3. Sud, D.; Mahajan, G.; Kaur, M. P.; Bioresour. Technol. 2008, 99, 6017.

4. Dorea, J. G.; Donangelo, C. M.; Clin. Nutrition 2006, 25, 369.

5. Baird, C.; Química Ambiental, 2a ed., Bookman: Porto Alegre, 2002.

6. Zambon, G. A.; Dissertação de Mestrado, Universidade Estadual de Campinas, Brasil, 2003.

7. Pansini, M.; Miner. Deposita 1996, 31, 563.
8. Goel, J.; Kadirvelu, K.; Rajagopal, C.; Garg, V. K.; J. Hazard. Mater. 2005, B125, 211.

9. Dias, J. M.; Alvim-Ferraz, M. C. M.; Almeida, M. F.; Utrilla, J. R.; Polo, M. S.; J. Environ. Manag. 2007, 85, 833.

10. Dwivedi, C. P.; Sahu, J. N.; Mohanty, C. R.; Mohan, B. R.; Meikap, B. C.; J. Hazard. Mater. 2008, 156, 596.

11. Dal Bosco, S. M.; Jimenez, R. S.; Vignado, C.; Fontana, J.; Geraldo, B.; Figueiredo, F. C. A.; Mandelli, D.; Carvalho, W. A.; Adsorption 2006, $12,133$.

12. Dal Bosco, S. M.; Jimenez, R. S.; Carvalho, W. A.; J. Colloid Interface Sci. 2005, 281, 424.

13. Carvalho, W. A.; Vignado, C.; Fontana, J.; J. Hazard. Mater. 2008, 153, 1240

14. Fontana, J., Riboldi, M. B.; Carvalho, W. A.; Vignado, C.; Adsorpt. Sci. Technol. 2007, 25, 673.

15. Stape, J. L.; Binkley. D.; Ryan, M. G.; Forest Ecol. Manag. 2008, 255, 920.

16. Carvalho W. A.; Bueno, C. I. C.; Quim. Nova 2007, 30, 1911.

17. Issabayeva. G.; Aroua, M. K.; Sulaiman, N. M.; J. Hazard. Mater. 2008, $155,109$.

18. Lv, L.; Zhang, Y.; Wang, K.; Ray, A. K.; Zhao, X. S.; J. Colloid Interface Sci. 2008, 325, 57.

19. Bohart, G. S.; Adams, E. Q.; J. Am. Chem. Soc. 1920, 42, 523.

20. Kumar, U.; Bandyopadhyay, M.; J. Hazard. Mater. 2006, 129, 253.

21. Bhakat, P. B.; Gupta, A. K.; Ayoob, S.; J. Hazard. Mater. 2007, 139, 286.

22. Hutchin, R. A.; Am. J. Chem. Eng. 1973, 80, 133.

23. Naiya, T. K.; Bhattacharya, A. K.; Das, S. K.; J. Colloid Interface Sci. 2009, 333, 14.

24. Pehlivan, E.; Altun, T.; Parlayici, S.; J. Hazard. Mater. 2009, 164, 982.

25. Sari, A.; Tuzen, M.; J. Hazard. Mater. 2009, 164, 1004. 


\section{REMOÇÃO DE CHUMBO(II) EM SISTEMAS CONTÍNUOS POR CARVÃO ATIVADO COM VAPOR}

Cristiane Imenes de Campos Bueno Zanin e Flávia Camargo Alves Figueiredo

Faculdade de Engenharia Química, Universidade Estadual de Campinas, CP 6066, 13083-970 Campinas - SP, Brasil

Wagner Alves Carvalho*

Centro de Ciências Naturais e Humanas, Universidade Federal do ABC, Rua Santa Adélia, 166, 09210-170 Santo André - SP, Brasil

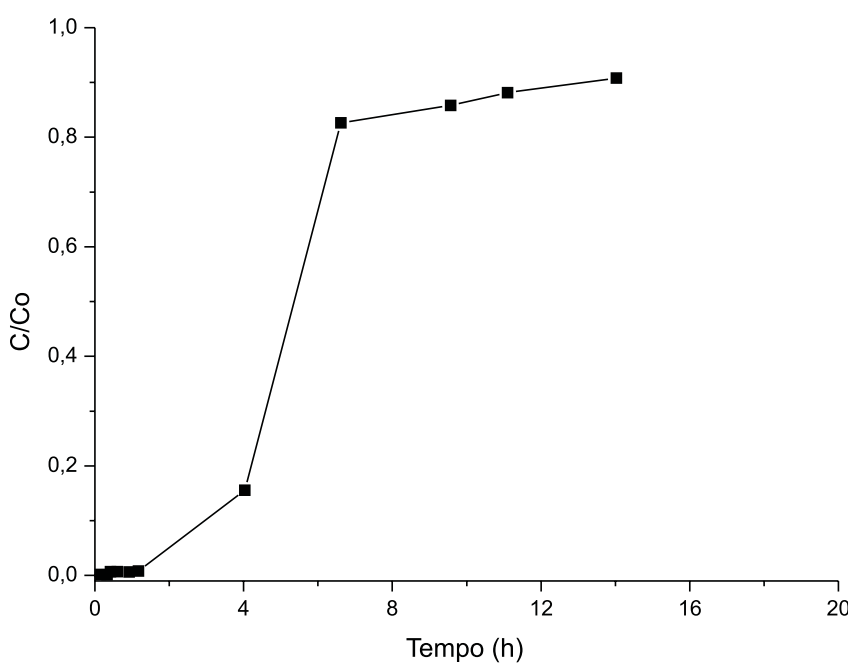

Figura 1S. Curva de ruptura para adsorção de Pb(II) $100 \mathrm{mg} \mathrm{L}^{-1} \mathrm{com} 0,3$ $g$ de carvão

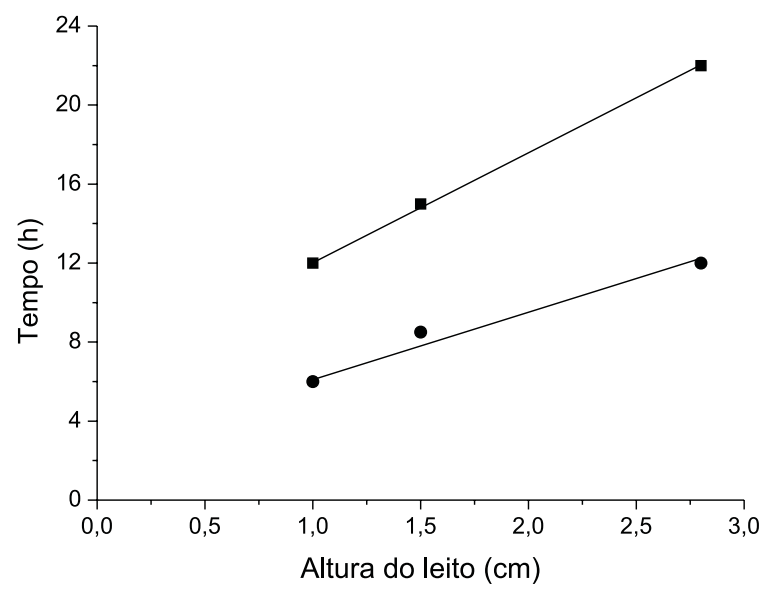

Figura 3S. Profundidade da coluna em função do tempo de serviço para concentração inicial de $\mathrm{Pb}(\mathrm{II}) 10 \mathrm{mg} \mathrm{L}^{-1}$ : (ロ) $C / C_{0}=0,5$, (o) $C_{B}=0,5 \mathrm{mg} \mathrm{L}^{-1}$

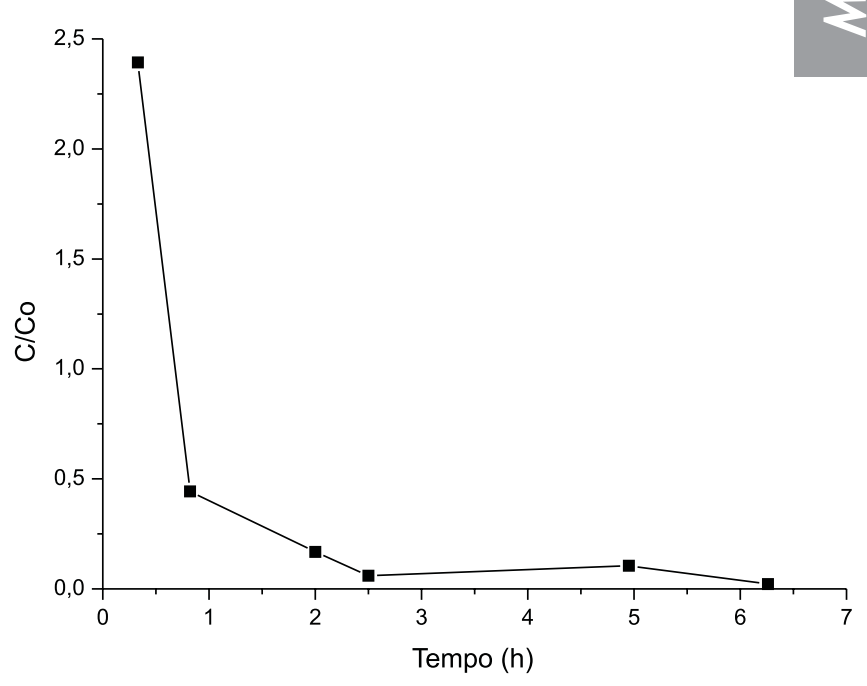

Figura 2S. Curva de ruptura para dessorção de $\mathrm{Pb}(\mathrm{II}) 100 \mathrm{mg} \mathrm{L}^{-1}$ com 0,3 g de carvão

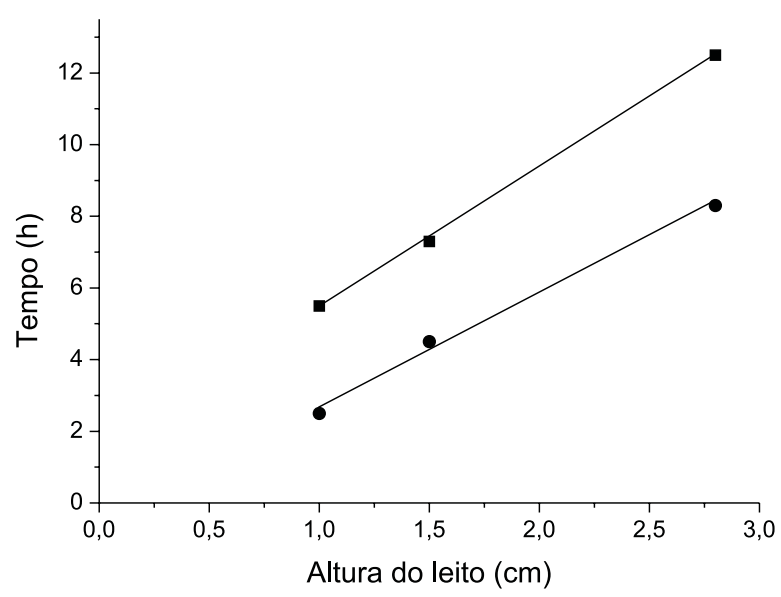

Figura 4S. Profundidade da coluna em função do tempo de serviço para concentração inicial de $\mathrm{Pb}(\mathrm{II}) 20 \mathrm{mg} \mathrm{L}^{-1}$ : (⿴) $C / C_{0}=0,5$, (o) $C_{B}=0,5 \mathrm{mg} \mathrm{L}^{-1}$ 


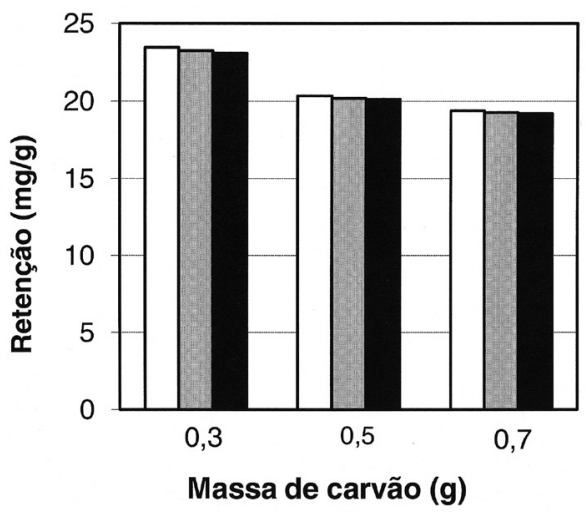

Figura 5S. Capacidade máxima de adsorção para a concentração inicial de Pb(II) de $10 \mathrm{mg} \mathrm{L}^{-1}$ nos três ciclos adsorção-dessorção (1 $1^{\circ}$. branco, $2^{\circ}$. cinza, $3^{\circ}$. preto)

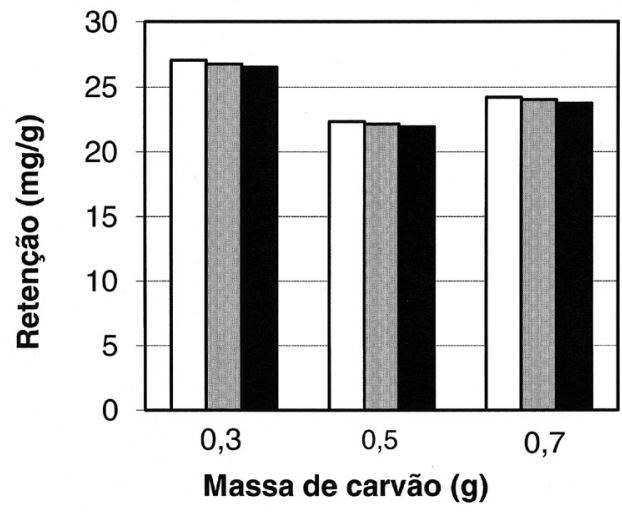

Figura 6S. Capacidade máxima de adsorção para a concentração inicial de $\mathrm{Pb}(\mathrm{II})$ de $20 \mathrm{mg} \mathrm{L}^{-1}$ nos três ciclos adsorção-dessorção $\left(1^{\circ}\right.$. branco, $2^{\circ}$. cinza, $3^{\circ}$. preto) 